\title{
TRATAMENTO DE CHORUME DE ATERRO SANITÁRIO POR FOTOCATÁLISE HETEROGÊNEA INTEGRADA A PROCESSO BIOLÓGICO CONVENCIONAL
}

\author{
Josmaria Lopes de Morais* \\ Centro Federal de Educação Tecnológica do Paraná, Av. Sete de Setembro, 3165, 80230-901 Curitiba - PR \\ Carla Sirtori e Patricio G. Peralta-Zamora \\ Departamento de Química, Universidade Federal do Paraná, CP19081, 81531-990 Curitiba - PR
}

Recebido em 26/8/04; aceito em 20/4/05; publicado na web em 10/8/05

\begin{abstract}
TREATMENT OF LANDFILL LEACHATES BY HETEROGENEOUS PHOTOCATALYSIS INTEGRATED TO A CONVENTIONAL BIOLOGICAL PROCESS. The chemical and microbiological decomposition of the garbage deposited in landfills leads to the generation of a dark and malodorous liquid residue that shows a chemical composition of extreme variability and complexity. When matured, the leachates show low biodegradability, which makes it difficult to treat by conventional biological processes. In this work a new strategy for the treatment of landfill leachates is proposed, involving a preliminary treatment by heterogeneous photocatalysis followed by an activated sludge system. The results demonstrate that photochemical treatments of 60 and 90 min significantly enhance the leachates' biodegradability favoring subsequent biological treatment. The biodegradability rate $(\mathrm{BOD} / \mathrm{COD})$ was also greatly enhanced.
\end{abstract}

Keywords: landfill leachates; heterogeneous photocatalysis; biodegradability.

\section{INTRODUÇÃO}

O chorume é um resíduo líquido de elevada carga orgânica e forte coloração, produzido pela decomposição química e microbiológica dos resíduos sólidos depositados em um aterro. A sua composição química apresenta grande variabilidade, uma vez que, além de depender da natureza dos resíduos depositados, da forma de disposição, manejo e da idade do aterro, é extremamente influenciada por fatores climáticos, dentre os quais pode-se destacar, a quantidade de chuva e a temperatura ${ }^{1,2}$.

De maneira geral, o chorume pode ser considerado como uma matriz de extrema complexidade, composta por quatro frações principais: matéria orgânica dissolvida (formada principalmente por metano, ácidos graxos voláteis, compostos húmicos e fúlvicos), compostos orgânicos xenobióticos (representados por hidrocarbonetos aromáticos, compostos de natureza fenólica e compostos organoclorados alifáticos), macrocomponentes inorgânicos (dentre os quais se destacam $\mathrm{Ca}, \mathrm{Mg}, \mathrm{Na}, \mathrm{K}, \mathrm{NH}_{4}^{+}, \mathrm{Fe}, \mathrm{Mn}, \mathrm{Cl}, \mathrm{SO}_{4}^{2-}$ e $\mathrm{HCO}_{3}^{-}$) e metais potencialmente tóxicos (ex. $\mathrm{Cd}, \mathrm{Cr}, \mathrm{Cu}, \mathrm{Pb}, \mathrm{Ni}$ e $\mathrm{Zn})^{3}$.

$\mathrm{O}$ impacto produzido pelo chorume no meio ambiente é bastante acentuado. Estudos recentes demonstram que efeitos adversos podem ser observados no solo, mesmo a distâncias superiores a $100 \mathrm{~m}$ do aterro, assim como alterações na biota aquática, principalmente nas imediações da descarga ${ }^{3,4}$. Por este motivo, a implementação de sistemas de coleta e tratamento é essencial.

No Brasil, a forma de tratamento mais comum é de natureza biológica. Este tipo de processo pode ser considerado eficiente no tratamento de chorume de aterro novo, rico em ácidos graxos voláteis e elevado valor de Demanda Bioquímica de Oxigênio (DBO) ${ }^{5,6}$. Entretanto, inúmeros problemas são detectados no tratamento de chorume de celas mais antigas (chorume maduro), o qual costuma apresentar reduzida disponibilidade de matéria orgânica biodegradável, altas concentrações de nitrogênio amoniacal, metais potencialmente tóxicos e substâncias húmicas ${ }^{7,8}$, além de outras es-

*e-mail: josmaria@cefetpr.br pécies complexas que resultam da condensação de núcleos aromáti$\cos ^{8,9}$. Em geral, admite-se que a permanência de compostos de alta massa molecular no lixiviado de aterro sanitário é um dos fatores responsáveis pela redução considerável de sua biodegradabilidade (determinada pela relação DBO/DQO) ao longo do tempo ${ }^{10}$.

Uma vez que não existem processos economicamente viáveis, que permitam reduzir todos os parâmetros ambientalmente relevantes da matriz de chorume, vários trabalhos recentes têm proposto o uso de processos integrados. Nestas propostas, processos físico-químicos de coagulação/floculação são aplicados de maneira preliminar, visando melhorar a eficiência de tratamentos biológicos subsequientes ${ }^{11,12}$. Também recentemente, processos oxidativos avançados têm sido integrados com processos biológicos convencionais, com resultados bastante promissores ${ }^{13,14}$.

Nos últimos anos, a fotocatálise heterogênea tem mostrado uma excelente potencialidade para a rápida degradação de substratos resistentes a outro tipo de tratamento ${ }^{15}$. O presente trabalho objetiva explorar esta característica, utilizando o processo fotocatalítico como sistema de pré-tratamento de amostras de chorume, visando aumentar sua biodegradabilidade aeróbia.

\section{PARTE EXPERIMENTAL}

\section{Substratos, reagentes}

As amostras de percolado de aterro sanitário foram coletadas das células mais antigas do Aterro Sanitário da Caximba (Curitiba-PR). O chorume foi caracterizado e armazenado em refrigerador a $4{ }^{\circ} \mathrm{C}$.

$\mathrm{TiO}_{2}$ (Degusa P25) e $\mathrm{ZnO}$ (Merse) foram utilizados sem nenhum tratamento prévio. Os outros reagentes usados foram de grau analítico.

\section{Caracterização do chorume}

O chorume utilizado nos estudos de degradação foi preliminarmente caracterizado, apresentando Demanda Química de Oxigê- 
nio (DQO) de $5500 \mathrm{mg} \mathrm{L}^{-1}$, Demanda Bioquímica de Oxigênio

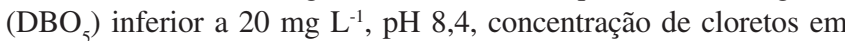
torno de $2500 \mathrm{mg} \mathrm{L}^{-1}$, concentração de nitrogênio amoniacal de $840 \mathrm{mg} \mathrm{L}^{-1}$ e condutividade de $25,0 \mathrm{mS} \mathrm{cm} \mathrm{cm}^{-1}$.

\section{Fotocatálise heterogênea}

Para realização do processo fotoquímico utilizou-se um reator fotoquímico cilíndrico de $100 \mathrm{~mL}$ de capacidade, equipado com sistema de refrigeração (empregando água), agitação magnética e sistema de oxigenação. Neste equipamento, a radiação ultravioleta foi proporcionada por lâmpada a vapor de mercúrio de $125 \mathrm{~W}$ (sem o bulbo protetor), inserida no interior do reator por meio de um tubo de quartzo. Inicialmente o semicondutor $(50 \mathrm{mg})$ era adicionado a $100 \mathrm{~mL}$ de chorume; a mistura obtida foi submetida a processo de agitação com o emprego de um agitador magnético (50 rpm) por 20 min e, em seguida irradiada. Oxigênio comercial foi borbulhado durante o processo fotoquímico, com vazão de aproxi-

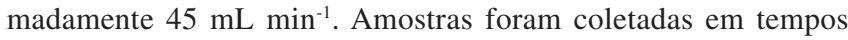
pré-determinados, filtradas em membrana Milipore ${ }^{\circledR}(0,45 \mu \mathrm{m}) \mathrm{e}$ submetidas a controle por espectroscopia UV-Vis, determinações DQO solúvel, DBO e fenóis totais.

\section{Processo biológico (lodos ativados em batelada)}

O processo foi desenvolvido empregando-se um conjunto formado por 8 reatores de bancada ( $1 \mathrm{~L}$ de capacidade), do tipo mistura completa.

O lodo ativado foi coletado junto à Estação Belém de Tratamento de Esgoto (Curitiba-PR), sendo utilizado de acordo com o esquema apresentado na Figura 1. A manutenção da viabilidade do lodo foi avaliada com determinações do Índice Volumétrico de Lodo (IVL) e observações microscópicas empregando lâminas de preparo simples. A eficiência da etapa de tratamento biológico foi avaliada por determinações de DQO solúvel. Todos os ensaios foram realizados em duplicata, sendo que os resultados apresentados correspondem à média aritmética das replicatas.
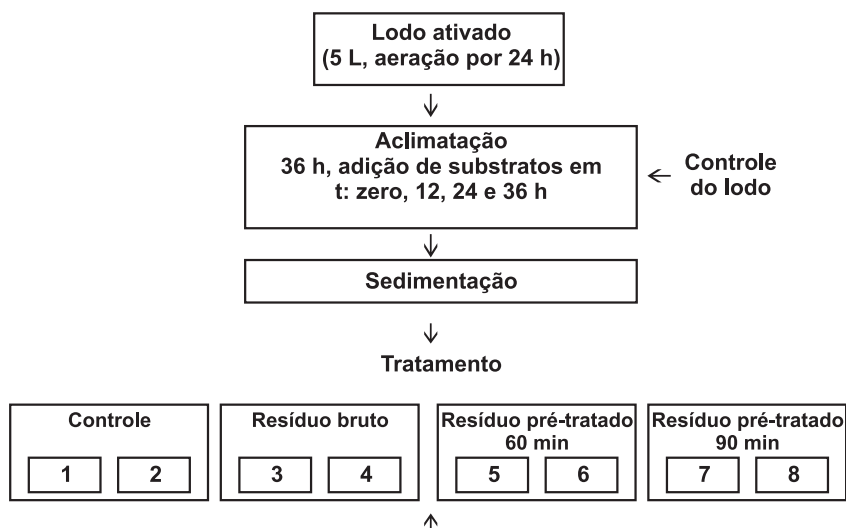

Controle de parâmetros do lodo e de degradação

Figura 1. Representação esquemática dos processos de adaptação e tratamento de chorume sem pré-tratamento e chorume tratado por processo fotoquímico em reatores de bancada tipo mistura completa

\section{Controles analíticos}

Tanto para caracterização das amostras de chorume, como para os controles de degradação fotoquímica foram utilizados os parâmetros DQO (Standart Methods 5220) e DBO (Standart Methods, 5210), determinados de acordo com procedimentos pa- drão ${ }^{16}$. As determinações (relativas) da presença de compostos fenólicos foram realizadas com base na reação da amostra com o reagente de Folin Ciocalteau, o que leva à formação de um complexo de coloração azul intensa, com absorbância máxima em 700 nm (Standart Methods 5550) ${ }^{16}$. Foram feitas determinações por cromatografia empregando-se um sistema cromatográfico Shimadzu modelo LC-10AD para cromatografia de permeação em gel ou exclusão molecular, equipado com duas colunas Tosoh do tipo TSKHXL. Os componentes da amostra foram detectados no eluato empregando-se refratometria diferencial, com detector Shimadzu modelo RID10A.

A degradação da matriz foi avaliada espectroscopicamente, monitorando-se a evolução da área espectral integrada de acordo com metodologia proposta Kwork et al. ${ }^{17}$. Foram realizados cálculos de área integrada entre 200 e $700 \mathrm{~nm}$, com emprego do programa Microcal Oringin ${ }^{\circledR}$. Absorbância em $410 \mathrm{~nm}$ foi empregada para se avaliar a remoção da cor do chorume (de marrom escurecido para amarelado), de acordo com metodologia descrita por Thomas et al. ${ }^{18}$.

O Índice Volumétrico de Lodo (IVL) foi calculado a partir da expressão abaixo apresentada, de acordo como procedimentos descritos na literatura ${ }^{16}$

$\operatorname{IVL}(\mathrm{mL} / \mathrm{g})=\frac{\mathrm{RS} 30(\mathrm{~mL} / \mathrm{L})}{\mathrm{SS}(\mathrm{g} / \mathrm{L})}$

sendo que RS 30 corresponde à Razão de Sedimentabilidade do Lodo e SS, ao teor de sólidos suspensos.

Estudos de microscopia ótica foram realizados empregando-se lâminas de preparo simples, a fresco, de acordo com procedimentos descritos na literatura ${ }^{19,20}$.

\section{RESULTADOS E DISCUSSÃO}

Inicialmente, a influência dos parâmetros $\mathrm{pH}$, natureza $(\mathrm{ZnO}$ ou $\mathrm{TiO}_{2}$ ) e massa do semicondutor foi avaliada por um sistema de planejamento fatorial de experimentos, utilizando-se a remoção de DQO como resposta analítica (Tabela 1). Os resultados indicaram uma discreta influência dos parâmetros estudados, com as melhores condições de degradação representadas por uma massa de 50

Tabela 1. Planejamento Fatorial $2^{3}$ com ponto central para sistemas de fotocatálise heterogênea, empregando $\mathrm{TiO}_{2}$ e $\mathrm{ZnO}$ livres

\begin{tabular}{|c|c|c|c|c|}
\hline Variável & \multicolumn{2}{|c|}{ Nível (-) } & Nível (•) & Nível (+) \\
\hline $\mathrm{pH}$ & \multicolumn{2}{|c|}{6,6} & 8,4 & 9,6 \\
\hline Massa (mg) & \multicolumn{2}{|c|}{25} & 50 & 100 \\
\hline Semicondutor & \multicolumn{2}{|c|}{$\mathrm{ZnO}$} & - & $\mathrm{TiO}_{2}$ \\
\hline \multirow[b]{2}{*}{ Experimento } & \multicolumn{3}{|c|}{ Variáveis } & \\
\hline & $\mathrm{pH}$ & Massa & $\mathrm{ZnO}, \mathrm{TiO}_{2}$ & $\begin{array}{c}\text { Redução de } \\
\text { DQO }(\%)\end{array}$ \\
\hline 1 & - & - & - & 13,7 \\
\hline 2 & - & - & + & 27,6 \\
\hline 3 & - & + & - & 25,2 \\
\hline 4 & - & + & + & 21,9 \\
\hline 5 & + & - & - & 22,8 \\
\hline 6 & + & - & + & 25,3 \\
\hline 7 & + & + & - & 37,3 \\
\hline 8 & + & + & + & 19,3 \\
\hline 9 & • & • & - & $36,2 \pm 8,0$ \\
\hline 10 & $\bullet$ & $\bullet$ & + & $39,3 \pm 3,4$ \\
\hline
\end{tabular}


mg de dióxido de titânio e pH natural das amostras de chorume $(8,4)$. Embora os resultados conseguidos com a utilização de óxido de zinco tivessem sido bastante similares, optou-se pelo dióxido de titânio em razão da sua maior inércia química. O ZnO apresenta uma elevada solubilidade em meio alcalino, o que pode levar à formação de zincatos, os quais, em função da sua toxicidade, influenciariam negativamente o processo biológico posterior.

Trabalhando-se em condições otimizadas, amostras de chorume foram submetidas ao processo fotocatalítico, obtendo-se os resultados apresentados na Figura 2. Os resultados demonstram modificações relevantes na matriz de chorume, as quais se traduzem em redução de cor (observação centrada em $410 \mathrm{~nm}$ ), DQO e área espectral. É importante salientar que para tratamentos de $60 \mathrm{~min}$, os parâmetros antes salientados foram reduzidos em 50, 40 e 35\%, respectivamente. $\mathrm{O}$ estudo realizado através do parâmetro fenóis totais demonstrou que o sistema atingiu degradações de $46 \%$ das formas fenólicas, em 60 min de tratamento.

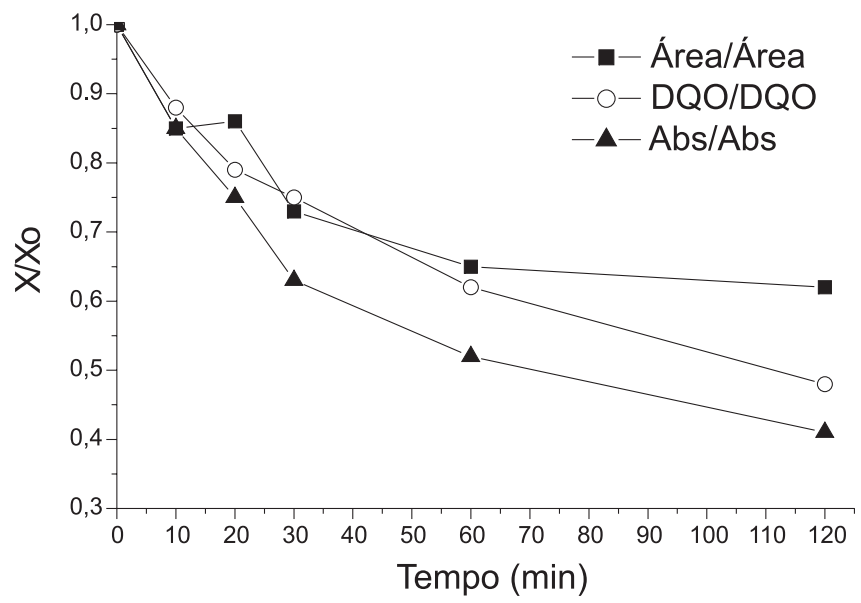

Figura 2. Evolução dos parâmetros de controle durante a degradação fotocatalítica da matriz de chorume. Área/Área representa a integração da área do espectro entre 200 e $700 \mathrm{~nm}, \mathrm{DQO} / \mathrm{DQO}$ a razão entre os valores de $D Q O$ solúvel e Abs/Abs, a absorbância em $410 \mathrm{~nm}$ e está relacionada à remoção da cor do chorume. Volume: $100 \mathrm{~mL}$; $\mathrm{pH} 8,4$; $\mathrm{TiO}_{2}: 50 \mathrm{mg}$

Antecedentes da literatura atribuem à matriz de chorume uma massa molecular aparente de 10.000 a $50.000 \mathrm{~g} \mathrm{~mol}^{-1}$, principalmente graças à contribuição de substâncias macromoleculares como lignina e ácidos húmicos ${ }^{21,22}$. Uma vez que estas substâncias são associadas à baixa biodegradabilidade do chorume, torna-se bastante interessante verificar o efeito do tratamento proposto na distribuição de massas moleculares da matriz em estudo. Acompanhando-se o processo por cromatografia de permeação em gel (Figura 3) foi possível verificar que espécies de elevada massa molecular, refratárias a tratamentos biológicos ${ }^{6}$, foram eficientemente fragmentadas, o que possivelmente seja bastante benéfico para o tratamento biológico posterior. Esta premissa foi confirmada nos estudos envolvendo a determinação da razão de biodegradabilidade $\left(\mathrm{DBO}_{5} / \mathrm{DQO}\right)$, apresentada na Tabela 2. Estes resultados demonstram que o tratamento fotoquímico permite uma degradação eficiente da fração orgânica mais resistente (representada pela DQO) e sua transformação em espécies de maior biodegradabilidade (representadas pela DBO). Considerando-se que um resíduo pode ser considerado biodegradável somente quando a razão de biodegradabilidade alcança valores em torno de $0,3^{23}$, podese supor que, tal como esperado, um tratamento fotoquímico de 60 min viabilize o tratamento biológico de uma matriz inicialmente não-biodegradável.

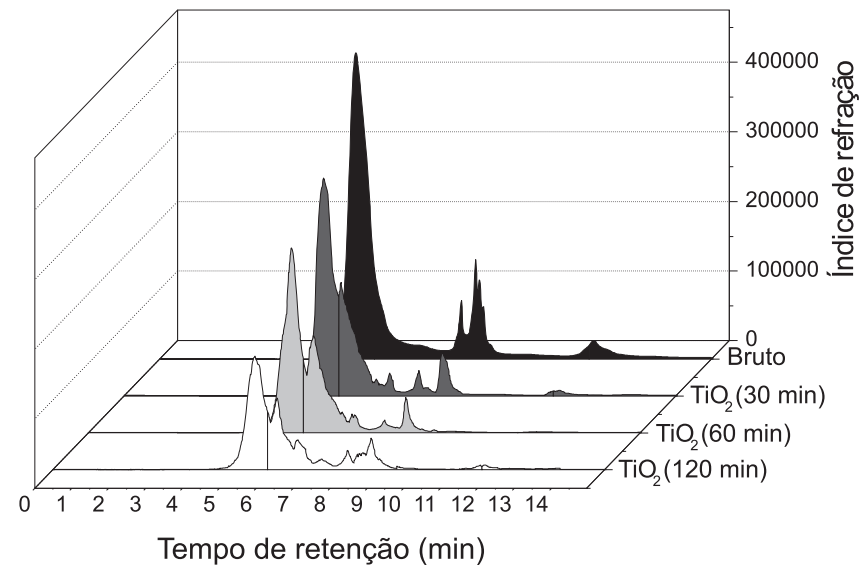

Figura 3. Evolução da distribuição de massas moleculares nas amostras de chorume submetidas a tratamento fotocatalítico, por cromatografia de permeação em gel

Para verificar esta premissa, foram realizados estudos de degradação pelo sistema biológico de lodos ativados, utilizando-se chorume bruto e pré-tratado fotoquimicamente. O dióxido de titânio presente no chorume fotoquimicamente tratado não foi retirado para a etapa biológica. Os resultados, apresentados como evolução da DQO e do índice volumétrico de lodo (IVL) na Figura 4, confirmaram as vantagens proporcionadas pelo uso de processos fotocatalíticos prévios. Na ausência de pré-tratamento, o processo biológico aplicado por $72 \mathrm{~h}$ permite uma redução de carga orgânica (DQO) da ordem de 20\%, enquanto que em amostras pré-tratadas a redução alcança valores próximos a $80 \%$.

Adicionalmente, o tratamento proposto melhora os parâmetros relacionados com a manutenção e viabilidade do lodo, aqui representados pelo índice volumétrico de lodo (Figura 4) e pelas análises microscópicas do lodo. De maneira geral, a qualidade do lodo em sistemas de lodos ativados pode ser avaliada por meio de sua sedimentabilidade, empregando-se o parâmetro IVL. A classificação referente a faixas típicas de IVL, proposta por von Sperlin ${ }^{24}$, corresponde a (em $\left.\mathrm{mL} \mathrm{g}^{-1}\right)$ : ótima (até 50$)$, boa (50-100), média (100-200), ruim (200-300) e péssima (>300). Foi possível observar que o tratamento fotoquímico prévio permite, na etapa de tratamento biológico, a manutenção do IVL na faixa considerada "boa", enquanto que a presença do chorume sem tratamento prévio no lodo dificulta a sedimentação da biomassa, proporcionando valo-

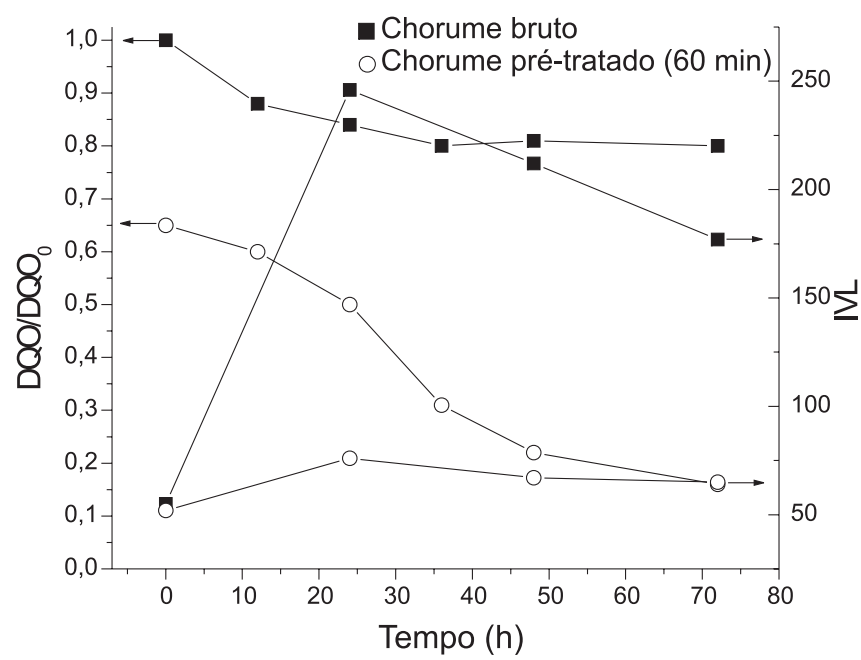

Figura 4. Evolução da DQO e do IVL durante tratamento biológico de amostras de chorume bruto e pré-tratado 
res de IVL superiores a $150 \mathrm{~mL} \mathrm{~g}^{-1}$, indo das condições "média" para "ruim", praticamente durante todo o período dedicado ao tratamento biológico. A consideração do comportamento dos sólidos no processo de lodos ativados é fundamental, pois a maior parte da DQO efluente do sistema é devida ao escape de sólidos em suspensão com o efluente durante a descarga do processo.

Tabela 2. Evolução da razão de biodegradabilidade durante o tratamento fotocatalítico das amostras de chorume

\begin{tabular}{lccc}
\hline Amostra & $\begin{array}{c}\mathrm{DBO}_{5} \\
\left(\mathrm{mg} \mathrm{O}_{2} \mathrm{~L}^{-1}\right)\end{array}$ & $\begin{array}{c}\mathrm{DQO} \\
\left(\mathrm{mg} \mathrm{O}_{2} \mathrm{~L}^{-1}\right)\end{array}$ & $\mathrm{DBO}_{5} / \mathrm{DQO}$ \\
\hline $\begin{array}{l}\text { Chorume } \\
\text { sem tratamento }\end{array}$ & $<20$ & $5500 \pm 400$ & 0,00 \\
$\mathrm{UV}^{-\mathrm{TiO}_{2}(60 \mathrm{~min})}$ & $1190 \pm 170$ & $3460 \pm 250$ & 0,34 \\
${\mathrm{UV}-\mathrm{TiO}_{2}(90 \mathrm{~min})}$ & $1312 \pm 97$ & $3132 \pm 320$ & 0,42 \\
\hline
\end{tabular}

Obs.: os valores indicados representam a média aritmética de 3 determinações.

Observações microscópicas diárias foram também realizadas (Figura 5), o que permitiu corroborar a melhor qualidade do lodo ativado nos processos que envolvem o tratamento biológico de chorume fotoquimicamente pré-tratado e contendo $\mathrm{TiO}_{2}$ em suspensão. Nestas amostras, e nas amostras de lodo controle, foram observados flocos bem estruturados de biomassa, enquanto que nas amostras envolvendo o tratamento de chorume bruto os flocos eram pequenos e compactos, o que é descrito por Jenkins et al. ${ }^{19}$ como "pin floc" ou "pinpoint floc", característicos de biomassa que não está assimilando a carga tóxica recebida.

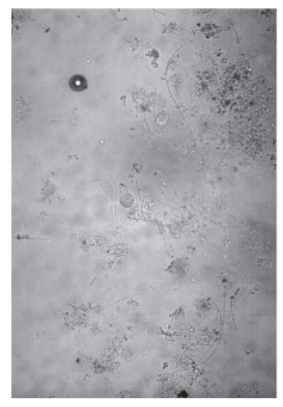

a

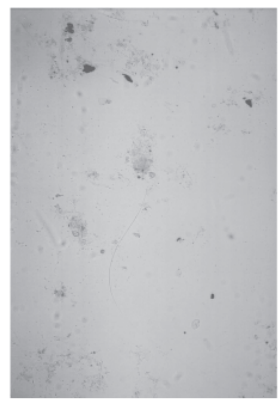

$\mathrm{b}$

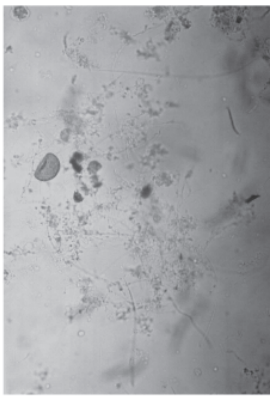

$\mathrm{c}$
Figura 5. Imagens microscópicas de lodo controle (a), lodo de tratamento de chorume bruto (b) e lodo de tratamento de chorume pré-tratado (c)

\section{CONCLUSÕES}

Em função dos antecedentes apresentados, é possível concluir que o tratamento fotoquímico proposto $\left(\mathrm{UV} / \mathrm{TiO}_{2}\right)$ induz importantes modificações na matriz de chorume, permitindo um significativo aumento da sua biodegradabilidade. Em geral, aumentos da ordem de 0,3 unidades foram observados na razão de biodegradabilidade $\left(\mathrm{DBO}_{5} / \mathrm{DQO}\right)$, em amostras submetidas a um tratamento prévio de $60 \mathrm{~min}$. Esse aumento da biodegradabilidade permitiu que o processo biológico posterior eliminasse aproximadamente $80 \%$ da carga orgânica representada pela DQO, enquanto que em sistemas sem pré-tratamento a remoção foi inferior a $20 \%$. A manutenção de melhores condições de sedimentabilidade do lodo, durante os processos de tratamento biológico, representa um importante indicativo adicional da potencialidade dos processos fotocatalíticos como sistemas de pré-tratamento. Dentro deste contexto, é importante salientar que os estudos de tratabilidade aeróbia de chorume, pré-tratado fotoquimicamente, têm demonstrado a inexistência de efeitos tóxicos associados ao $\mathrm{TiO}_{2}$, assim como o favorecimento da manutenção da viabilidade do lodo, provavelmente em função do semicondutor servir como suporte da biomassa.

Uma vez que o sistema proposto foi estudado em escala de bancada, a avaliação de custos operacionais é bastante dificultada, principalmente quando se deseja realizar extrapolações para escalas maiores. De qualquer forma, o custo operacional está representado principalmente pelo consumo energético associado ao processo fotoquímico, o qual corresponde a aproximadamente 1,25 $\mathrm{kWh} \mathrm{L}^{-1}$, na configuração estudada.

\section{REFERÊNCIAS}

1. Im, J. H.; Woo, H. J.; Choi, M. W.; Han, K. B.; Kim, C.W.; Water Res. 2001, 35, 2403.

2. Kjeldesen, P.; Barlaz, M. A.; Rooker, A. P.; Baun, A.; Ledin, A.; Christensen, T.H.; Crit. Rev. Environ. Sci. Technol. 2002, 32, 297; El Fadel, M; Douseid, E.; Chahine, W.; Alaylic, B.;Waste Management 2002, 22, 269.

3. Christensen, T. H.; Bjerg, P.; Jensen, D. L.; Christensen J. B.; Christensen, A.; Baun, A.; Albrecgtsen, H. J.; Heron, G.; Appl. Geochem. 2001, 16, 659.

4. Baun, A.; Reitzel, L. A.; Ledin, A.; Christensen, T. H.; Bjerg, P. L.; J. Contam. Hydrol. 2003, 2, 1916.

5. Amokrane, A.; Comel, C.; Veron, J.; Water Res. 1997, 11, 2775; Loukidou, M.; Zouboulis, A. I.; Environ. Pollut. 2001, 111, 273.

6. Zouboulis, A. I.; Chai, X. L; Katsoyiannis, I. A.; J. Environ. Management 2004, 70, 35

7. Ozturk, I.; Altinbas, M.; Koyuncu, I.; Arikan, O.; Gomec-Yangin, C.; Waste Management 2003, 23, 441.

8. Christensen, J. B.; Jensen, D. L.; Gron, C.; Filip, Z.; Christensen, T. H.; Water Res. 1998, 32, 125.

9. Tatsi, A. A.; Zouboulis, A. I.; Matis, K. A.; Samaras, P.; Chemosphere 2003, 53, 737; Nascimento Filho, I.; Von Mühlen, C.; Caramão, E. B.; Quim. Nova 2001, 24, 554

10. Manning, D. A. C.; Bewsher, A.; J. Chromatogr. 1997, 770, 203; Martienssen, M.; Schöps, R.; Water Res. 1997, 31, 1164.

11. Sletten, R. S.; Benhamin, M. M.; Horng, J. J.; Ferguson, J. H.; Water Res. 1995, 29, 2376; Lin, S. H.; Chang, C.; Water Res. 2000, 34, 4243.

12. Silva, A. C.; Dezotti, M.; Sant'anna, G.L.; Chemosphere 2004, 55, 207; Wang, P.; Zhang, Z.; Lin, Y. J.; Deng, N.-S.; Tao, T.; Zhuo, K.; J. Hazard. Mater. 2002, 2887, 1.

13. Koh, I-O.; Chen-Hamacher, X.; Hicke, K.; Thiermann, W.; J. Photochem. Photobiol. 2004, 162, 261.

14. Lopez, A.; Pagano, M.; Volpe, A.; Di Pinto, A. C.; Chemosphere 2004, 54, 1005 .

15. Nogueira, R. F. P.; Jardim, W. F.; Quim. Nova, 1998, 21, 69; Pirkanniemi, K.; Sillanpaa, M.; Chemosphere 2002, 48, 1047; Parra, S.; Malato, S.; Pulgarin, C.; Catal. Today 2002, 76, 3019.

16. APHA; Standard Methods for the Examination of Water and Wastewater, $19^{\text {th }}$ ed.; Ed. Amer. Publ. Health Ass. nº5550p, 1995.

17. Kwork, Y. K.; Wong, H. Y.; Lau, K. W.; J. Complexity 2001, 17, 773.

18. Thomas, O.; El Khorassani, H.; Touraud, E.; Bitar, M.; Talanta 1999, 50, 743.

19. Jenkins, D.; Richard, M. G.; Daigger, G. T.; Manual on the Causes and Control of Activated Sludge Bulking and Foaming, Ed. Lewis Publishers: Michigan, 1993.

20. Vazzoler, R. F.; Microbiologia de Lodos Ativados, Série Manuais CETESB, 1989.

21. Borges, R. M. H.; Silva, L. R.; Campos, J. C.; VI Simpósio Ítalo Brasileiro de Engenharia Sanitária e Ambiental, Vitória, Brasil, 2002.

22. Yu, G.; Zhu, W.; Yang, Z.; Chemosphere 1998, 38, 487.

23. Karrer, N. J.; Ryhiner, G.; Hinzle, E.; Water Res. 1997, 31, 1013.

24. von Sperling, M.; Revista Engenharia Sanitária e Ambiental 2001, 6, 149. 